\title{
Recyclable PUFs: Logically Reconfigurable PUFs
}

\author{
Stefan Katzenbeisser ${ }^{1}$, Ünal Koçabas ${ }^{1}$, Vincent van der Leest ${ }^{2}$, \\ Ahmad-Reza Sadeghi ${ }^{3}$, Geert-Jan Schrijen ${ }^{2}$, Heike Schröder ${ }^{1}$, \\ and Christian Wachsmann ${ }^{1}$ \\ 1 Technische Universität Darmstadt (CASED), Germany \\ \{katzenbeisser, busch\}@seceng. informatik.tu-darmstadt.de, \\ \{unal.kocabas, christian.wachsmann\}@trust.cased.de \\ 2 Intrinsic-ID, Eindhoven, The Netherlands \\ \{vincent.van.der.leest, geert.jan.schrijen\}@intrinsic-id.com \\ 3 Technische Universität Darmstadt and Fraunhofer SIT Darmstadt, Germany \\ ahmad.sadeghi@trust. cased.de
}

\begin{abstract}
We introduce the concept of Logically Reconfigurable Physical Unclonable Functions (LR-PUFs). In contrast to classical Physically Unclonable Functions (PUFs) LR-PUFs can be dynamically 'reconfigured' after deployment such that their challenge/response behavior changes in a random manner. To this end, we amend a conventional PUF with a stateful control logic that transforms challenges and responses of the PUF. We present and evaluate two different constructions for LR-PUFs that are simple, efficient and can easily be implemented. Moreover, we introduce a formal security model for LR-PUFs and prove that both constructions are secure under reasonable assumptions. Finally, we demonstrate that LR-PUFs enable the construction of securely recyclable access tokens, such as electronic tickets: LR-PUFs enhance security against manipulation and forgery, while reconfiguration allows secure re-use of tokens for subsequent transactions.
\end{abstract}

\section{Introduction}

In the last decades we are witnessing a rapid development and enhancement as well as an evolution of information technologies: On the one hand, computing and communication devices tend to become increasingly smaller and physically highly integrated. On the other hand, the growing usage and interconnection of millions of devices processing sensitive information raises many new trust and security challenges. Hence enabling technologies that can uniquely identify an (embedded) device and use the corresponding identity as a trust anchor in higher level security architectures are highly desirable. Although modern cryptography provides many useful tools for authentication and secure channels, it cannot guarantee the device's integrity, in particular in presence of hardware attacks.

In this context, Physically Unclonable Functions (PUFs) seem to be promising primitives that aim to exploit (random) physical variations to extract unique features of the underlying hardware to uniquely identify a device. The assumed properties of PUFs such as unclonability, unpredictability and tamper-evidence 
make them very appealing for deployment in cryptographic applications. Since their introduction by Pappu 3839], PUFs have been proposed for secure generation and storage of strong cryptographic keys (see, e.g., 52 26]), and for emerging hardware-entangled cryptography [3], where the security of the cryptographic scheme is based on the physical properties of PUFs instead of mathematical problems. Moreover, today, there are already PUF-based security products aimed for the market (e.g., RFID, IP-protection, anti-counterfeiting solutions) [51 16].

So far, most existing PUFs exhibit a static behavior while a variety of applications greatly benefits from the availability of PUFs whose characteristics can be changed dynamically, i.e., reconfigured, after deployment: For instance, PUF-based key storage [52 26] and PUF-based cryptographic primitives [3] may require that previous secrets derived from the PUF cannot be retrieved any more. Another example are solutions to prevent downgrading of software [20] by binding the software to a certain hardware configuration, e.g., a PUF, which require the PUF behavior to be irreversibly altered upon installation of a software update. Moreover, when PUF-based wireless access token:11 (e.g., [0|49|3742 51]) are re-used/recycled, the new users of the token shall not be able to retrieve access rights and/or to obtain privacy-sensitive information of the previous users of the token (see, e.g., [53174]).

Unfortunately, all known implementations of physically reconfigurable PUFs rely on optical mechanisms, reconfigurable hardware (i.e., FPGAs), or novel memory technologies 20], which all have serious drawbacks in practice. In particular, optical PUFs cannot easily be integrated into integrated circuits and require expensive and error-prone evaluation equipment while FPGA-based solutions cannot be realized with non-reconfigurable hardware (e.g., ASICs) that is commonly used in practice [29].

Our goal and contributions. In this paper, we propose Logically Reconfigurable PUFs (LR-PUFs), an alternative construction to physically reconfigurable PUFs. LR-PUFs augment a physical PUF with a stateful control logic that changes the challenge/response behavior of the LR-PUF according to its internal state 2 In particular, our contributions are as follows:

- New constructions: We propose two different constructions for logically reconfigurable PUFs (LR-PUFs). Our performance measurements show that the implementation overhead of the logical reconfiguration on top of a physical PUF is rather small.

- Security model: We introduce a formal security model for LR-PUFs and prove that both of our constructions are secure. More precisely, we show that, when instantiated by an appropriate physical PUF under reasonable assumptions,

${ }^{1}$ PUFs provide a lightweight and cost-effective solution to the problem of detecting counterfeit or cloned access tokens (e.g., RFID-based electronic tickets) by cryptographically binding a user's access rights to the physical characteristics of the token.

2 A similar concept has been independently proposed by Lao et al. 22. However, they do not provide a (formal) security model and do not discuss the adversary model and assumptions underlying their constructions. 
our LR-PUFs can achieve both forward- and backward-unpredictability: The former assures that responses measured before the reconfiguration event are invalid thereafter, while the latter assures that an adversary with access to a reconfigured PUF cannot estimate the PUF behavior before reconfiguration.

- Applications: We demonstrate how LR-PUFs could be deployed for re-usable (recyclable) access tokens, such as electronic transit tickets, and discuss other envisaged applications of LR-PUFs.

Note that, although the constructions of LR-PUFs as proposed in this paper seem to be similar to Controlled PUFs [11, LR-PUFs and Controlled PUFs have very different objectives: In contrast to Controlled PUFs, LR-PUFs do not aim to prevent modeling attacks on PUFs but provide a practical way to enable reconfigurability for existing, typically static PUF constructions. We will elaborate on this aspect in Section 3 .

Outline. The rest of the paper is structured as follows: After providing background information on Physically Unclonable Functions (PUFs) in Section 2, we present the concept of Logically Reconfigurable PUFs (LR-PUFs) in Section 3 . We show two concrete LR-PUF constructions in Section 4, describe their implementation and evaluate their performance in Section 5] and formally prove their security in Section [6. In Section [7] we show how LR-PUFs could be used to realize recyclable access tokens and discuss several other potential use cases of LR-PUFs. Finally, we conclude in Section 8 .

\section{Background: Physically Unclonable Functions (PUFs)}

A Physically Unclonable Function (PUF) is a noisy function that is embedded into a physical object, e.g., an integrated circuit 392. When queried with a challenge $w$, a PUF generates a response $y \leftarrow \operatorname{PUF}(w)$ that depends on both $w$ and the unique device-specific intrinsic physical properties of the object containing PUF(). Since PUFs are subject to noise (e.g., environmental variations), they return slightly different responses when queried with the same challenge multiple times.

In literature, PUFs are typically assumed to be robust, physically unclonable, unpredictable and tamper-evident, and several approaches to heuristically quantify and formally define their properties have been proposed (see [2] for a comprehensive overview). Robustness means that, when queried with the same challenge multiple times, the same PUF will always return the same response. Physical unclonability means that it is infeasible to produce two PUFs that cannot be distinguished based on their challenge/response behavior, which cannot be achieved by (cryptographic) algorithms. Unpredictability requires that it is infeasible to predict the PUF response to a given unknown challenge, even if the PUF can be adaptively queried for a certain number of times. Since this is the most interesting property for cryptographic applications of PUFs [2], we will formally define unpredictability later, when we prove the security of our LR-PUF constructions. Tamper-evidence means that any attempt to physically access the 
PUF irreversibly changes its challenge/response behavior. This is an important issue for practical deployment since it allows the detection of invasive hardware attacks, to which embedded devices are typically exposed to in practice.

A broad variety of different PUF constructions exists (see 29] for an overview). The most appealing ones for integration into electronic circuits are electronic PUFs. The most prominent examples of electrical PUFs include delay-based PUFs that exploit race conditions (arbiter PUFs [23 37/27]) and frequency variations (ring oscillator PUFs [124830]) that can be found in integrated circuits; memory-based PUFs that are based on the instability of volatile memory cells like SRAM [1415], flip-flops [28 24] and latches [4719]; and coating PUFs [50], which are based on the capacitance caused by a special dielectric coating applied to the chip that houses the PUF.

Note that the amount of unique responses of a memory-based PUF is limited by the number of its memory cells. Moreover, it has been shown that most delay-based PUFs are subject to model building attacks that allow simulating the PUF in software (see, e.g., [233727/41]). To counter this problem, additional primitives must be used: Controlled PUFs [11 use cryptography in hardware to hide the actual response of the underlying PUF, which prevents model building attacks. However, this requires the link between the PUF and the crypto component as well as the crypto component itself to be protected against invasive and/or side channel attacks.

\section{Logically Reconfigurable PUFs}

A logically reconfigurable PUF (LR-PUF) is a PUF whose challenge/response behavior depends on both the physical properties of the PUF and the logical state maintained by a control logic, as shown in Figure 1(a) The challenge/response behavior of the LR-PUF can be dynamically changed after it has been deployed by updating its state.

\subsection{System Model}

An LR-PUF combines a conventional physically unclonable function PUF () and a control logic circuit. As shown in Figure 1(b), the control logic maintains a $S$, which is stored in non-volatile memory, provides an algorithm query ${ }_{S}()$ for querying, and $r \operatorname{cnf}()$ for reconfiguring the LR-PUF. The algorithm query $\mathrm{S}_{S}()$ consists of an input transformation function $\operatorname{mapin}_{S}()$ and an output transformation function mapout ${ }_{S}()$ : query m $_{S}(x)$ computes $w \leftarrow \operatorname{mapin}_{S}(c)$, evaluates $y \leftarrow \operatorname{PUF}(w)$, and returns $r \leftarrow \operatorname{mapout}_{S}(y)$. The algorithm implementing $\operatorname{rcnf}()$ reconfigures the LR-PUF by changing the current state $S$ to a new independent state $S^{\prime} \leftarrow \operatorname{rcnf}()$.

Note that the generic LR-PUF construction depicted in Figure 1(b) can be seen as a generalization of controlled PUFs [11]. Controlled PUFs aim to hide the challenge/response behavior of the underlying PUF to the adversary to prevent model building attacks [1141 by applying an appropriate mapin() and/or 


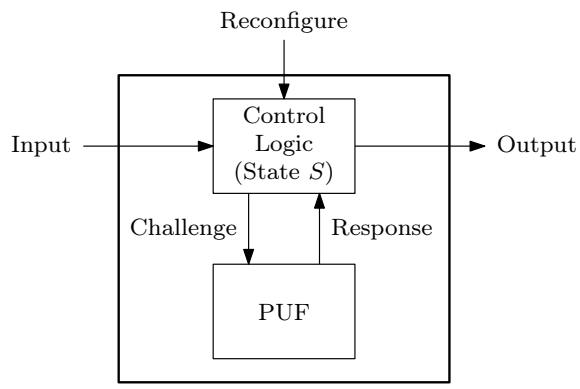

(a) LR-PUF concept

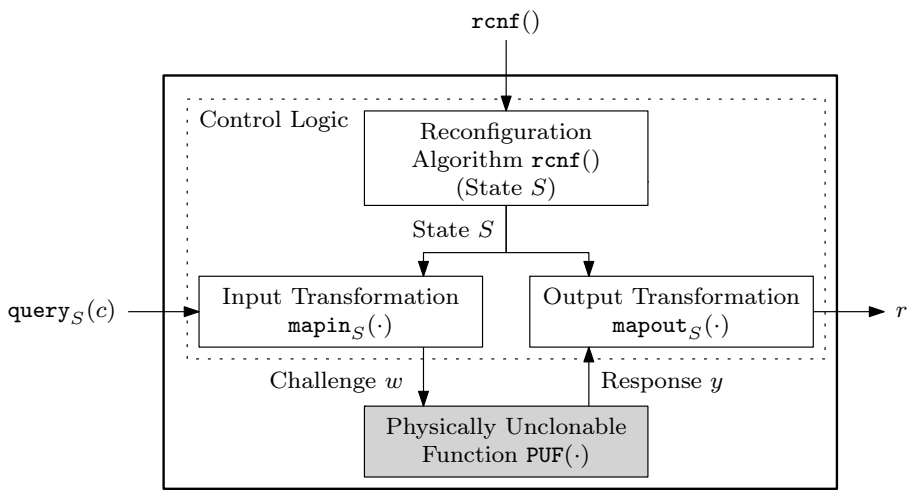

(b) Generic LR-PUF construction

Fig. 1. Logically Reconfigurable PUFs: Concept and generic construction

mapout() function. In contrast, LR-PUFs aim to enable reconfigurability for conventional non-reconfigurable PUFs after they have been deployed by entangling an updatable state with the challenges and/or responses of the underlying PUF.

\subsection{Assumptions and Adversary Model}

We assume that the underlying PUF is physically unclonable and unpredictable (see Section 2). The algorithms mapin(), mapout(), and $\operatorname{rcnf}()$ are publicly known. Moreover, the adversary $\mathcal{A}$ is assumed to know the current and all previous states $S$ of the LR-PUF, e.g., by performing hardware attacks like side-channel or invasive attacks. However, we assume that $\mathcal{A}$ cannot force the control logic to set the LR-PUF state to a specific value, i.e., $\mathcal{A}$ cannot change the state $S$ of the LR-PUF to a value of its choice (e.g., an old LR-PUF state).

For this, it must be assured that (1) $\operatorname{rcnf}()$ cannot be manipulated such that it generates predictable states, and that (2) the non-volatile memory cells storing the LR-PUF state cannot be set to specific values (e.g., by hardware attacks). The first requirement can be achieved by implementing the reconfiguration function using a fault injection aware design at a reasonable performance 
penalty 311. Moreover, although fault injection attacks against non-volatile memory (e.g., EEPROM or Flash) have been shown [44, it seems to be difficult in practice to perform invasive attacks that change the content of specific nonvolatile memory cells without affecting the content of the surrounding cells [45]. Hence, in practice it should be infeasible for an adversary to write a specific value (e.g., an old LR-PUF state) into the non-volatile memory of the LR-PUF. In particular, due to the increasing complexity of modern embedded systems and the fact that technology nodes are progressively getting smaller, the amount of precision and the quality of the equipment required to successfully perform such attacks renders them uneconomical in most practical applications (e.g., electronic ticketing).

\subsection{Security Objectives}

As pointed out in Section 2, physical unclonability and unpredictability are fundamental security requirements for PUF-based applications. Ideally, an LR-PUF should resemble a physically reconfigurable PUF. This implies that it should be infeasible for an adversary $\mathcal{A}$ to predict the response to a challenge of an LRPUF for some state, even if $\mathcal{A}$ knows the responses to this challenge of the same LR-PUF but for other (e.g., old) states. Here, we must distinguish between the case where $\mathcal{A}$ aims to predict the responses of the LR-PUF for the current state (e.g., to forge a PUF response in an authentication protocol) or for a previous LR-PUF state (e.g., to recover an old key bound to the previous LR-PUF state). Moreover, in most applications of reconfigurable PUFs, it must be infeasible to set the state of the LR-PUF to a specific value, which would allow resetting the LR-PUF to a previous state and may help the adversary to predict LR-PUF responses. We first informally summarize the security requirements of LR-PUFs below and later give formal definitions and proofs for two different LR-PUF constructions in Section 6 .

- Backward unpredictability: The adversary $\mathcal{A}$ cannot predict the response of the LR-PUF for a previous state $S$ (i.e., before reconfiguration) to a challenge that has not been queried for the previous state, even if $\mathcal{A}$ knows an adaptively chosen set of challenge/response pairs of the LR-PUF for the previous state and can adaptively obtain challenge/response pairs of the LR-PUF for the current state.

- Forward unpredictability: The adversary $\mathcal{A}$ cannot predict the response of an LR-PUF for the current state $S$ to a challenge that has not yet been queried for the current state, even if $\mathcal{A}$ knows an adaptively chosen set of challenge/response pairs of the LR-PUF for the previous state and can adaptively obtain challenge/response pairs of the LR-PUF for the current state (except for the challenge in question).

- Non-resettability: The adversary cannot set the state of the LR-PUF to a specific value. 


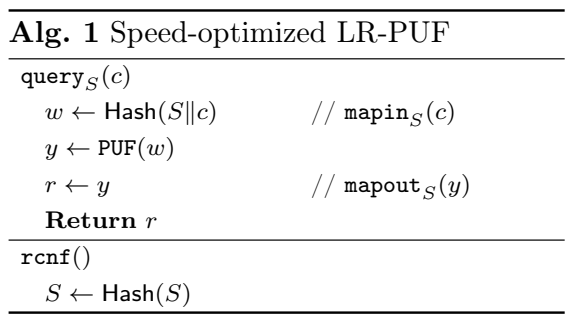

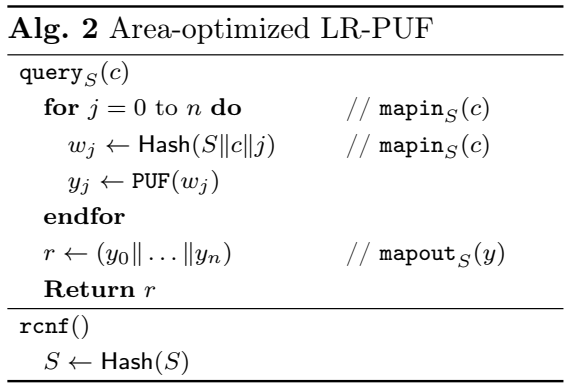

\section{Constructions}

In this section, we present two instantiations of our generic LR-PUF construction described in Section 3 . The first construction is optimized for the fast generation of responses, while the second construction aims for the area constraints of low-cost devices (e.g., RFID chips) and provides a tradeoff between response generation time and the amount of area required.

\subsection{Speed-Optimized LR-PUF Construction}

Our first construction uses a PUF with a large challenge and a large response space and implements the control logic based on a single collision-resistant hash function. The challenge space must be large since otherwise it may be possible to create a complete challenge/response pair (CRP) database, which allows simulating the PUF. A large response space is a fundamental security requirement in many applications such as PUF-based identification/authentication [52 26] and hardware-entangled cryptography 3, where it is crucial that the PUF response to a formerly unknown challenge can be guessed with negligible probability only.

Our first construction is specified in Algorithm 1 and works as follows: When challenged with query $(c)$, the control logic computes $w \leftarrow \operatorname{Hash}(S \| c)$ and returns $y \leftarrow \operatorname{PUF}(w)$, i.e., $\operatorname{mapin}_{S}(c):=\operatorname{Hash}(S \| c)$ and $\operatorname{mapout}_{S}(y):=y$. To reconfigure the LR-PUF, $\operatorname{rcnf}()$ sets the LR-PUF state to $S \leftarrow \operatorname{Hash}(S)$.

Since most PUF constructions that support a large challenge space (e.g., arbiter PUFs 233727]) typically have only a small response space, several of these PUFs can be evaluated in parallel on the same challenge, which, however, significantly increases the amount of area required for their implementation. The collision-resistance property of the hash function assures the unpredictability property of the LR-PUF (see Section 2), as we will show later in the formal security analysis. Note that the LR-PUF state is just used to parameterize the hash function and thus needs not to be secret. Hence, to reconfigure the LRPUF it is sufficient to hash the previous LR-PUF state to obtain a new and independent state (assuming the hash function implements a random oracle). 


\subsection{Area-Optimized LR-PUF Construction.}

Our first LR-PUF construction described in Section 4.1 typically requires multiple parallel PUFs. Hence, we propose a second construction using just one single PUF that is evaluated sequentially $n$ times to generate an $n$ bit LR-PUF response, providing a tradeoff between area consumption and response generation speed. The intuition of this second construction is very similar as for the speedoptimized construction described in Section 4.1. Note that the underlying PUF must be queried with different challenges to generate a large response consisting of different (ideally) independent bits. This can be achieved by including a counter $j$ as additional input to the hash function that now generates a sequence of PUF challenges $w_{j}$ from the LR-PUF challenge $c$ and the current LR-PUF state $S$. The corresponding PUF responses $y_{j}$ are then concatenated to form the response $r$ of the LR-PUF.

Our second construction is specified in Algorithm 2 and works as follows: On query $(c)$, the control logic computes $\operatorname{mapin}_{S}(c)$ as $w_{j} \leftarrow$ Hash $(S\|w\| j)$ for $j \in\{0, \ldots, n\}$, evaluates $y_{j} \leftarrow \operatorname{PUF}\left(w_{j}\right)$, and $\operatorname{mapout}_{S}()$ returns $r \leftarrow\left(y_{0}\|\ldots\| y_{n}\right)$. To reconfigure the LR-PUF, $\operatorname{rcnf}()$ sets the LR-PUF state to $S \leftarrow \operatorname{Hash}(S)$.

\section{Implementation and Performance Evaluation}

Both constructions presented in Section 4 are based on PUFs with a large challenge space. The only existing electronic PUFs that provide this feature seem to be arbiter PUFs [2313]. The hash function of the control logic can be implemented efficiently by using a lightweight block cipher.

We implemented a prototype of both of our LR-PUF constructions on a Xilinx Spartan-6 FPGA board. We instantiated the underlying PUF based on arbiter PUFs that support 64 bit challenges and generate 1 bit responses, following the approach in 46. The hash function of the control logic is based on the PRESENT block cipher [5] in Davies-Meyer mode [21]. Both resulting LR-PUF implementations use 80 bit challenges and generate 64 bit responses.

Table 1. Performance results of the LR-PUF constructions presented in Section 4

\begin{tabular}{|l|c|c|c|c|}
\hline Optimization & Response time & \multicolumn{4}{|c|}{ Area consumption in slices (gate equivalents) } \\
& in clock cycles & Control logic & Arbiter PUF & Total \\
\hline \hline Speed & 1069 & $166(1162 \mathrm{GE})$ & $4288(29056 \mathrm{GE})$ & $4454(30218 \mathrm{GE})$ \\
\hline Area & 64165 & $358(2506 \mathrm{GE})$ & $67(454 \mathrm{GE})$ & $425(2960 \mathrm{GE})$ \\
\hline
\end{tabular}

We evaluated our implementation with regard to response generation speed and area consumption. Our results are summarized in Table 1. The second column shows the time in number of clock cycles required to compute an LR-PUF response $r$. The remaining columns show the number of slices and gate equivalents (GE) required to implement the control logic, the PUF, and the overall construction. The area estimation does not include the non-volatile memory for 
storing the LR-PUF state, which cannot be implemented on FPGA. Our results show that the area-optimized construction requires only about $10 \%$ of the area of the speed-optimized construction but is 60 times slower.

Note that our implementation is meant to demonstrate the feasibility of our approach and to obtain performance results. Due to the technical constraints of FPGAs, our implementation does not cover the non-volatile memory for storing the LR-PUF state, which is emulated by providing the state as an input to the FPGA. Moreover, our implementation is based on arbiter PUFs, which do not have the unpredictability property [4] that is required for the security of our constructions. To securely implement our LR-PUF constructions, the underlying PUF must be unpredictable (e.g., a Controlled PUF [11 can be used) and the non-volatile memory and control logic should be protected against fault-injection attacks, e.g., by applying the techniques described in [311.

\section{Security Definitions and Evaluation}

In this section we formally define the LR-PUF security properties of forward-and backward-unpredictability and show that both are fulfilled by the constructions proposed in Section 4. To this end, we first formalize the security property of unpredictability of a standard PUF.

Unpredictability of a PUF. Along the lines of [2] we define unpredictability of a PUF in terms of an unpredictability game between an adversary $\mathcal{A}$ and a challenger $\mathcal{C}$. $\mathcal{A}$ is first given a PUF and is allowed to query it at most $q$ times. This step allows to model adversaries that are able to "learn" challenge/response pairs (CRPs) either by direct physical access to the interface of the PUF or by eavesdropping on messages containing PUF challenges and responses. At the end of the game, $\mathcal{A}$ is required to output a (non-trivial) valid pair of a PUF challenge and response.

\section{Unpredictability Game of a PUF}

Setup: The challenger $\mathcal{C}$ issues the PUF to the adversary $\mathcal{A}$.

Queries: Proceeding adaptively, $\mathcal{A}$ queries the PUF at most $q$ times on challenges $w_{i}$ (for $\left.1 \leq i \leq q\right)$. For each query, $y_{i} \leftarrow \operatorname{PUF}\left(w_{i}\right)$ is given to $\mathcal{A}$.

Output: Eventually, $\mathcal{A}$ outputs a challenge/response pair $\left(w^{*}, y^{*}\right)$.

Let $Q$ denote the set of all challenges issued by $\mathcal{A}$. We say that $\mathcal{A}$ wins the game, if $y^{*}$ is a valid $\operatorname{PUF}$ response to $\operatorname{PUF}\left(w^{*}\right)$ and $w^{*} \notin Q$. Conversely, a $\mathrm{PUF}$ is unpredictable, if no efficient adversary $\mathcal{A}$ is able to win the game with significant success probability:

Definition 1. A PUF is $(q, \varepsilon)$-unpredictable, if no probabilistic polynomial adversary $\mathcal{A}$ that makes at most $q$ queries to the LR-PUF is able to win the unpredictability game with a probability greater than $\varepsilon$. 
Backward- and Forward-Unpredictability of an LR-PUF. We define backwardand forward-unpredictability in terms of a two-stage game between an adversary $\mathcal{A}$ and a challenger $\mathcal{C}$. In the first stage, $\mathcal{A}$ is given oracle access (i.e., access to the interface) of the LR-PUF, from which $\mathcal{A}$ can obtain challenge/response pairs (CRPs) at will. This stage models the ability of $\mathcal{A}$ to obtain challenges and responses (with respect to a fixed internal LR-PUF state) by passive eavesdropping. We also give $\mathcal{A}$ access to the internal LR-PUF state $S$ in order to model hardware attacks against the LR-PUF implementation. Once $\mathcal{A}$ has learned enough CRPs, the challenger performs the reconfiguration operation and finally gives $\mathcal{A}$ oracle access to the reconfigured LR-PUF such that $\mathcal{A}$ can obtain CRPs of the reconfigured LR-PUF. At the end of the game, $\mathcal{A}$ outputs a prediction $\left(c^{*}, r^{*}\right)$ of an LR-PUF challenge/response pair.

More formally, $\mathcal{A}=\left(\mathcal{A}_{L}, \mathcal{A}_{C}\right)$ consists of two probabilistic polynomial time algorithms, where $\mathcal{A}_{L}$ interacts with the LR-PUF before reconfiguration and $\mathcal{A}_{C}$ thereafter. $\mathcal{A}$ engages in the following experiment:

\section{Backward- and Forward-Unpredictability Game of an LR-PUF}

Setup: The challenger $\mathcal{C}$ sets up an LR-PUF by choosing a random state $S$, which is given to the adversary $\mathcal{A}=\left(\mathcal{A}_{L}, \mathcal{A}_{C}\right)$.

Phase I: $\mathcal{A}_{L}$ is allowed to call query ${ }_{S}()$ of the LR-PUF up to $q_{L}$ times. At the end of phase I, $\mathcal{A}_{L}$ stops and outputs a log file $s t$ that is used as input to $\mathcal{A}_{C}$. We denote with $Q_{L}$ the set of challenges issued by $\mathcal{A}_{L}$ during phase I.

Reconfiguration: $\mathcal{C}$ reconfigures the LR-PUF by calling $r \mathrm{cnf}()$, which updates the internal LR-PUF state to $S^{\prime}$.

Phase II: $\mathcal{A}_{C}$ is initialized with log file st from $\mathcal{A}_{L}$ and the LR-PUF state $S^{\prime}$. $\mathcal{A}_{C}$ is allowed to query the reconfigured LR-PUF query ${ }_{S^{\prime}}()$ up to $q_{C}$ times on arbitrary challenges. We denote with $Q_{C}$ the set of challenges issued by $\mathcal{A}_{C}$ during phase II.

Output: $\mathcal{A}_{C}$ outputs a challenge/response pair $\left(c^{*}, r^{*}\right)$ of the LR-PUF.

Depending on whether we consider backward- or forward-unpredictability, we can state different conditions of an adversary being successful: $\mathcal{A}$ wins the backward-unpredictability game if $r^{*}$ is a valid LR-PUF response to query ${ }_{S^{\prime}}\left(c^{*}\right)$ and $c^{*} \notin Q_{C}$. Thus, once the LR-PUF has been reconfigured, the adversary cannot output a (non-trivial) challenge/response pair for the reconfigured LRPUF. Conversely, $\mathcal{A}$ wins the forward-unpredictability game if $r^{*}$ is a valid LRPUF response to query ${ }_{S}\left(c^{*}\right)$ and $c^{*} \notin Q_{L}$. Thus, an adversary, who has access to a reconfigured LR-PUF cannot predict (non-trivial) responses of the LR-PUF before reconfiguration happened. We say that an LR-PUF is backward- (resp. forward-) unpredictable, if no efficient adversary $\mathcal{A}$ is able to win the game with significant success probability:

Definition 2 (Backward- and Forward-Unpredictability). An LR-PUF is $\left(q_{L}, q_{C}, \varepsilon\right)$-backward unpredictable (resp. forward-unpredictable), if no probabilistic polynomial adversary $\mathcal{A}$ that makes at most $q_{L}$ queries in phase $I$ and at most $q_{C}$ queries in phase II, is able to win the backward-unpredictability (resp. forward-unpredictability) game with a probability greater than $\varepsilon$. 
Both constructions of Section 4 achieve backward- and forward- unpredictability:

Proposition 1. The speed-optimized LR-PUF construction shown in Section 4.1 is $\left(q_{L}, q_{C}, \varepsilon\right)$-backward unpredictable (resp. forward-unpredictable), if Hash () is collision-resistant and the underlying PUF is $\left(q_{L}+q_{C}, \varepsilon\right)$-unpredictable.

Proposition 2. The area-optimized LR-PUF construction shown in Section 4.2 is $\left(q_{L}, q_{C}, \varepsilon\right)$-backward unpredictable (resp. forward-unpredictable), if Hash () is collision-resistant and the underlying PUF is $\left(n\left(q_{L}+q_{C}\right), \varepsilon\right)$-unpredictable.

The proofs of both propositions follow from the standard reductionist approach and can be found in the full version of this paper [18. In particular, we show that any adversary $\mathcal{A}$ against the LR-PUF can be converted into an adversary $\mathcal{B}$ that either breaks the collision resistance of the hash function or the unpredictability of the underlying physical PUF. To this end, $\mathcal{B}$ simulates $\mathcal{A}$ : Whenever $\mathcal{A}$ makes an LR-PUF query, $\mathcal{B}$ simulates this query by help of his PUF oracle, i.e., $\mathcal{B}$ transforms the challenges received from $\mathcal{A}$ by using the (known) internal LRPUF state, queries the physical PUF on the transformed challenge and returns the obtained response to $\mathcal{A}$. Once the simulation stops, it can easily be seen that either a hash collision or a valid prediction of a challenge/response pair of the physical PUF can be extracted from $\mathcal{A}$ 's output.

\section{Applications}

\subsection{LR-PUF-Based Authentication Tokens}

Electronic payment and ticketing systems have been gradually introduced in many countries over the past few years (see, e.g., [35/7/33]). Typically, these systems are using RFID-enabled tokens and provide different types of electronic transit tickets. Given the typically large number of tickets used in an electronic transit ticket system and the costs per token (typically between 1-3 Euro), from an economic perspective it may be worthwhile to consider recycling of RFIDbased tickets. In fact, some systems (e.g., the Dutch transportation system [36]) allow recharging RFID-based tickets with money and to returning used tickets to the vendor with possible restitution of preloaded money left on the ticket. Moreover, many U.S. and European governments make manufacturers and importers of electronic products responsible for the disposal of their products when discarded by the consumer (see, e.g., [69]). In this context, recyclable tokens can help to save waste disposal costs and to reduce the amount of electronic waste. In this section, we discuss how LR-PUFs could be used to enhance the security of electronic ticketing and payment systems while at the same time enabling secure and privacy-preserving recycling of used RFID-tickets.

There are several proprietary solutions for electronic tickets in practice. Most of them are based on widely used RFID tokens, where the most prominent example is the MiFare family produced by NXP Semiconductors [34]. There are several hard- and software attacks against MiFare Classic tokens [32]43]10, which use a 
proprietary encryption algorithm that has been completely broken [8]. However, other MiFare products are claimed not to be affected. A recent attack on MiFare Classic $4 \mathrm{~K}$ chipcards concerns the Dutch electronic payment and transit ticket system [36]: Using a MiFare compatible card reader and a software from the Internet, an average user can add debit to his RFID-based transit ticket without being detected [54 25].

In this context, PUFs could provide a cost-effective security mechanism: Authentication based on PUFs can prevent copying and manipulating the information (i.e., the debit of the RFID-based ticket and/or the user's rights) by cryptographically binding this data to the physical characteristics of the underlying RFID chip. Existing PUF-based authentication schemes (see, e.g., 40153742 151]) typically assume each device, i.e., each RFID-based token $\mathcal{T}$, to be equipped with a PUF, whereas the verifier $\mathcal{V}$ maintains a database $\mathcal{D}$, i.e., a set if challenge/response pairs (CRPs) of each ticket. In the authentication protocol, $\mathcal{V}$ chooses a random challenge from $\mathcal{D}$ and sends it to $\mathcal{T}$, which then returns some response. $\mathcal{V}$ accepts if the response of $\mathcal{T}$ matches the one in $\mathcal{D}$.

Using LR-PUFs instead of non-reconfigurable PUFs would allow for cost-effective, secure and privacy-preserving recycling of RFID-based tickets: By reconfiguring the LR-PUF all information and access rights bound to $\mathcal{T}$ are securely "erased", which cannot be achieved with non-reconfigurable PUFs. However, reconfiguring the LR-PUF invalidates the CRP database $\mathcal{D}$ of $\mathcal{V}$, which means that after each reconfiguration of $\mathcal{T}$ a new CRP database must be established. To counter this problem, $\mathcal{V}$ could know the LR-PUF state $S$ of each token and maintain a CRP database $\mathcal{D}^{\prime}$ of the PUF underlying the LR-PUF, which can be seen as the "authentication secrets" of the token. This is common in ticketing applications because usually the verifier is the ticket issuer who typically knows the authentication secrets of all tokens. Since the algorithms of the control unit, i.e., the input and output transition functions mapin() and mapout(), respectively, and the state update algorithm $\operatorname{rcnf}()$, are publicly known, $\mathcal{V}$ could use $\mathcal{D}^{\prime}$ to recompute the LR-PUF response for any state of $\mathcal{T}$ and compare it to the response sent by $\mathcal{T} . \mathcal{V}$ accepts if the response of $\mathcal{T}$ matches the one recomputed based on $\mathcal{D}^{\prime}$ and $S$.

\subsection{Other Applications Envisaged}

Many airlines have started to move from paper-based tickets to electronic tickets. However, they still print luggage tags, which are increasingly equipped with disposable RFID chips. The purpose of these chips is to ease the tracking of individual luggage in the process of loading. However, RFID-enabled labels could be read out even without visual contact. This may allow several attacks ranging from copying luggage tags to smuggle in additional luggage in the name of another passenger. Moreover, RFID-enabled luggage tags may disclose personal information on their owner (e.g., name, number of luggage pieces, luggage weight), which could be used to track the user on the airport or provide useful information to luggage thieves. To solve these problems, travellers could purchase or rent a more powerful LR-PUF-enabled RFID token that is put into the 
luggage or that could even be embedded into new generations of suitcases. Each time the traveller checks in, his RFID-based tag is reconfigured by the airline attendant, which securely erases the previous information stored on it. This prevents tracking the traveler for more than one flight and impedes misrouting of luggage due to old travel information. Further, to avoid illegitimate tracking of travellers, the RFID-enabled luggage tag could be reconfigured or temporarily disabled once the passenger leaves the baggage claim area.

One can find many other applications that could take advantage of LR-PUFs. Examples include, secure deletion and/or update of cryptographic secrets in PUF-based key storage [52 26] and hardware-entangled cryptography [3], where the reconfiguration of the PUF ensures that old secrets cannot be retrieved any more. Another example are solutions to prevent downgrading of software [20] by binding the software to the PUF, where reconfiguring the PUF invalidates the old software version such that only the latest version can be used.

\section{Conclusion}

In this paper, we have proposed the concept of logically reconfigurable PUFs, which utilize a control logic to enable dynamic reconfigurability for existing, typically static PUFs. We introduced two different constructions to realize LRPUFs: Our first construction is optimized for response generation speed, while our second construction aims for resource-constrained embedded devices (like RFID tags). Furthermore, we have shown that both constructions achieve the security properties of backward- and forward unpredictability, which are two desirable properties in the context of PUF-based cryptographic applications like key storage, device identification, and hardware-entangled cryptography. Finally, we showed how LR-PUFs could be applied in the context of recyclable (access) tokens to enhance the security properties of existing solutions while providing a means for secure recycling of PUF-based access tokens.

Acknowledgements. We thank our anonymous reviewers for their helpful comments, Patrick Koeberl and Jérôme Quevremont for several useful discussions on hardware attacks and use cases, and Timm Korte for providing us his implementation of PRESENT. This work has been supported in part by the European Commission under grant agreement ICT-2007-238811 UNIQUE.

\section{References}

1. Akdemir, K.D., Wang, Z., Karpovsky, M.G., Sunar, B.: Design of cryptographic devices resilient to fault injection attacks using nonlinear robust codes. In: Fault Analysis in Cryptography (2011)

2. Armknecht, F., Maes, R., Sadeghi, A.R., Standaert, F.X., Wachsmann, C.: A formal foundation for the security features of physical functions. In: IEEE Symposium on Security and Privacy, pp. 397-412. IEEE Computer Society, Los Alamitos (2011) 
3. Armknecht, F., Maes, R., Sadeghi, A.R., Sunar, B., Tuyls, P.: Memory leakageresilient encryption based on physically unclonable functions. In: Matsui, M. (ed.) ASIACRYPT 2009. LNCS, vol. 5912, pp. 685-702. Springer, Heidelberg (2009)

4. Armknecht, F., Sadeghi, A.R., Visconti, I., Wachsmann, C.: On RFID privacy with mutual authentication and tag corruption. In: Zhou, J., Yung, M. (eds.) ACNS 2010. LNCS, vol. 6123, pp. 493-510. Springer, Heidelberg (2010)

5. Bogdanov, A., Knudsen, L., Leander, G., Paar, C., Poschmann, A., Robshaw, M., Seurin, Y., Vikkelsoe, C.: PRESENT: An ultra-lightweight block cipher. In: Paillier, P., Verbauwhede, I. (eds.) CHES 2007. LNCS, vol. 4727, pp. 450-466. Springer, Heidelberg (2007)

6. Californians Against Waste: E-waste laws in other states (April 2011), http://www. cawrecycles.org/issues/ca_e-waste/other_states

7. Calypso Networks Association: Website (April 2011), http: //www. calypsonet-asso.org/

8. Courtois, N.T., Nohl, K., O'Neil, S.: Algebraic attacks on the Crypto-1 stream cipher in MiFare Classic and Oyster Cards. Cryptology ePrint Archive, Report 2008/166 (2008)

9. European Commission: Waste electrical and electronic equipment website (April 2011), http://ec.europa.eu/environment/waste/weee/index_en.htm

10. Garcia, F.D., de Koning Gans, G., Muijrers, R., van Rossum, P., Verdult, R., Schreur, R.W., Jacobs, B.: Dismantling MIFARE classic. In: Jajodia, S., Lopez, J. (eds.) ESORICS 2008. LNCS, vol. 5283, pp. 97-114. Springer, Heidelberg (2008)

11. Gassend, B., Clarke, D., van Dijk, M., Devadas, S.: Controlled physical random functions. In: Computer Security Applications Conference, pp. 149-160. IEEE Computer Society, Los Alamitos (2002)

12. Gassend, B., Clarke, D., van Dijk, M., Devadas, S.: Silicon physical random functions. In: ACM Conference on Computer and Communications Security (ACM CCS), pp. 148-160 (2002)

13. Gassend, B., Lim, D., Clarke, D., van Dijk, M., Devadas, S.: Identification and authentication of integrated circuits. Concurrency and Computation: Practice and Experience 16(11), 1077-1098 (2004)

14. Guajardo, J., Kumar, S.S., Schrijen, G.J., Tuyls, P.: FPGA intrinsic PUFs and their use for IP protection. In: Paillier, P., Verbauwhede, I. (eds.) CHES 2007. LNCS, vol. 4727, pp. 63-80. Springer, Heidelberg (2007)

15. Holcomb, D.E., Burleson, W.P., Fu, K.: Initial SRAM state as a fingerprint and source of true random numbers for RFID tags. In: Conference on RFID Security (RFIDSec) (2007)

16. Intrinsic ID: Product webpage (April 2011), http://www.intrinsic-id.com/products.htm

17. Juels, A.: RFID security and privacy: A research survey. Journal of Selected Areas in Communication 24(2), 381-395 (2006)

18. Katzenbeisser, S., Ünal Kocabas, van der Leest, V., Sadeghi, A.R., Schrijen, G.J., Schröder, H., Wachsmann, C.: Recyclable PUFs: Logically reconfigurable PUFs (full version) (June 2011), http://www.trust.cased.de/

19. Kumar, S., Guajardo, J., Maes, R., Schrijen, G.J., Tuyls, P.: Extended abstract: The butterfly PUF protecting IP on every FPGA. In: IEEE Workshop on Hardware-Oriented Security and Trust (HOST), pp. 67-70 (2008)

20. Kursawe, K., Sadeghi, A.R., Schellekens, D., Tuyls, P., Scoric, B.: Reconfigurable physical unclonable functions - Enabling technology for tamper-resistant storage. In: IEEE International Workshop on Hardware-Oriented Security and Trust (HOST), pp. 22-29. IEEE Computer Society, San Francisco (2009) 
21. Lai, X., Massey, J.: Hash functions based on block ciphers. In: Rueppel, R.A. (ed.) EUROCRYPT 1992. LNCS, vol. 658, pp. 55-70. Springer, Heidelberg (1993)

22. Lao, Y., Parhi, K.K.: Novel reconfigurable silicon unclonable functions. In: Workshop on Foundations of Dependable and Secure Cyber-Physical Systems (FDSCPS) (April 11, 2011)

23. Lee, J.W., Lim, D., Gassend, B., Suh, G.E., van Dijk, M., Devadas, S.: A technique to build a secret key in integrated circuits for identification and authentication application. In: Symposium on VLSI Circuits, pp. 176-179 (2004)

24. van der Leest, V., Schrijen, G.J., Handschuh, H., Tuyls, P.: Hardware intrinsic security from D flip-flops. In: ACM Workshop on Scalable Trusted Computing (ACM STC), pp. 53-62 (2010)

25. Letter from Dutch minister on OV-chipkaart, https://zoek. officielebekendmakingen.nl/dossier/32440/kst-23645-415. $\mathrm{html}$

26. Lim, D., Lee, J.W., Gassend, B., Suh, G.E., van Dijk, M., Devadas, S.: Extracting secret keys from integrated circuits. IEEE Transactions on VLSI Systems 13(10), 1200-1205 (2005)

27. Lin, L., Holcomb, D., Krishnappa, D.K., Shabadi, P., Burleson, W.: Low-power sub-threshold design of secure physical unclonable functions. In: ACM/IEEE International Symposium on Low Power Electronics and Design (ISLPED), pp. 43-48 (2010)

28. Maes, R., Tuyls, P., Verbauwhede, I.: Intrinsic PUFs from flip-flops on reconfigurable devices. In: Workshop on Information and System Security (WISSec), p. 17 (2008)

29. Maes, R., Verbauwhede, I.: Physically unclonable functions: A study on the state of the art and future research directions. In: Sadeghi, A.R., Naccache, D. (eds.) Towards Hardware-Intrinsic Security. Information Security and Cryptography, pp. 3-37. Springer, Heidelberg (2010)

30. Maiti, A., Casarona, J., McHale, L., Schaumont, P.: A large scale characterization of RO-PUF. In: IEEE Symposium on Hardware-Oriented Security and Trust (HOST), pp. 94-99 (2010)

31. Monnet, Y., Renaudin, M., Leveugle, R.: Designing resistant circuits against malicious faults injection using asynchronous logic. IEEE Trans. Comput. 55, 1104-1115 (2006), http://dx.doi.org/10.1109/TC.2006.143

32. Nohl, K., Plötz, H.: MiFare - Little security despite obscurity (2007), http://events.ccc.de/congress/2007/Fahrplan/events/2378.en.html

33. NXP Semiconductors: MiFare applications (April 2008), http://www.mifare.net/applications/

34. NXP Semiconductors: MiFare smartcard ICs (February 2011), http://www.mifare.net/products/smartcardics/

35. Octopus Holdings: Website (April 2011), http://www.octopus.com.hk/en/

36. OV-Chipkaart: Website (April 2011), http://www.ov-chipkaart.nl/

37. Öztürk, E., Hammouri, G., Sunar, B.: Towards robust low cost authentication for pervasive devices. In: IEEE International Conference on Pervasive Computing and Communications (PERCOM 2008). IEEE Computer Society, Los Alamitos (2008)

38. Pappu, R.S.: Physical one-way functions. Ph.D. thesis, Massachusetts Institute of Technology (March 2001)

39. Pappu, R.S., Recht, B., Taylor, J., Gershenfeld, N.: Physical one-way functions. Science 297, 2026-2030 (2002) 
40. Ranasinghe, D.C., Engels, D.W., Cole, P.H.: Security and privacy: Modest proposals for low-cost RFID systems. In: Auto-ID Labs Research Workshop (September 2004)

41. Rührmair, U., Sehnke, F., Sölter, J., Dror, G., Devadas, S., Schmidhuber, J.: Modeling attacks on physical unclonable functions. In: ACM conference on Computer and communications security (ACM CCS), pp. 237-249 (2010)

42. Sadeghi, A.R., Visconti, I., Wachsmann, C.: PUF-enhanced RFID security and privacy. In: Workshop on Secure Component and System Identification, SECSI (2010)

43. Schreur, R.W., van Rossum, P., Garcia, F., Teepe, W., Hoepman, J.H., Jacobs, B., de Koning Gans, G., Verdult, R., Muijrers, R., Kali, R., Kali, V.: Security flaw in MiFare Classic (March 2008), http://www.sos.cs.ru.nl/applications/rfid/pressrelease.en.html

44. Skorobogatov, S.: Semi-invasive attacks - A new approach to hardware security analysis. Technical Report UCAM-CL-TR-630, University of Cambridge, $15 \mathrm{JJ}$ Thomson Avenue, Cambridge CB03 0FD, UK (April 2005)

45. Skorobogatov, S.: Local heating attacks on Flash memory devices. In: IEEE International Workshop on Hardware-Oriented Security and Trust (HOST 2009), pp. 1-6. IEEE, Los Alamitos (July 27, 2009)

46. Soybali, M., Ors, B., Saldamli, G.: Implementation of a PUF circuit on an FPGA. In: IFIP International Conference on New Technologies Mobility and Security (2011)

47. Su, Y., Holleman, J., Otis, B.: A 1.6pJ/bit $96 \%$ stable chip-ID generating circuit using process variations. In: IEEE International Solid-State Circuits Conference (ISSCC), pp. 406-611 (2007)

48. Suh, G.E., Devadas, S.: Physical unclonable functions for device authentication and secret key generation. In: Design Automation Conference, pp. 9-14 (2007)

49. Tuyls, P., Batina, L.: RFID-tags for anti-counterfeiting. In: Pointcheval, D. (ed.) CT-RSA 2006. LNCS, vol. 3860, pp. 115-131. Springer, Heidelberg (2006)

50. Tuyls, P., Schrijen, G.-J., Škorić, B., van Geloven, J., Verhaegh, N., Wolters, R.: Read-proof hardware from protective coatings. In: Goubin, L., Matsui, M. (eds.) CHES 2006. LNCS, vol. 4249, pp. 369-383. Springer, Heidelberg (2006)

51. Verayo, Inc.: Product webpage (April 2011), http://www . verayo.com/product/products.html

52. Škorić, B., Tuyls, P., Ophey, W.: Robust key extraction from physical uncloneable functions. In: Ioannidis, J., Keromytis, A.D., Yung, M. (eds.) ACNS 2005. LNCS, vol. 3531, pp. 407-422. Springer, Heidelberg (2005)

53. Weis, S.A., Sarma, S.E., Rivest, R.L., Engels, D.W.: Security and privacy aspects of low-cost radio frequency identification systems. In: Hutter, D., Müller, G., Stephan, W., Ullmann, M. (eds.) Security in Pervasive Computing. LNCS, vol. 2802, pp. 5059. Springer, Heidelberg (2004)

54. Wikipedia: OV-Chipkaart, http://en.wikipedia.org/wiki/OV-chipkaart 